\title{
Evaluating the exit pressure method for measurements of normal stress difference at high shear rates
}

Dahang Tang, Flávio H. Marchesini, Ludwig Cardon, and Dagmar R. D’hooge

Citation: Journal of Rheology 64, 739 (2020); doi: 10.1122/1.5145255

View online: https://doi.org/10.1122/1.5145255

View Table of Contents: https://sor.scitation.org/toc/jor/64/3

Published by the The Society of Rheology

\section{ARTICLES YOU MAY BE INTERESTED IN}

reptate rheology software: Toolkit for the analysis of theories and experiments Journal of Rheology 64, 709 (2020); https://doi.org/10.1122/8.0000002

Elastoviscoplastic rheology and aging in a simplified soft glassy constitutive model Journal of Rheology 64, 723 (2020); https://doi.org/10.1122/1.5140465

Evaluating predictability of various constitutive equations for MAOS behavior of entangled polymer solutions Journal of Rheology 64, 673 (2020); https://doi.org/10.1122/1.5139685

Strain shifts under stress-controlled oscillatory shearing in theoretical, experimental, and structural perspectives: Application to probing zero-shear viscosity

Journal of Rheology 63, 863 (2019); https://doi.org/10.1122/1.5111358

Stress-controlled oscillatory flow initiated at time zero: A linear viscoelastic analysis

Journal of Rheology 64, 545 (2020); https://doi.org/10.1122/1.5127827

Questioning a fundamental assumption of rheology: Observation of noninteger power expansions Journal of Rheology 64, 625 (2020); https://doi.org/10.1122/1.5130707

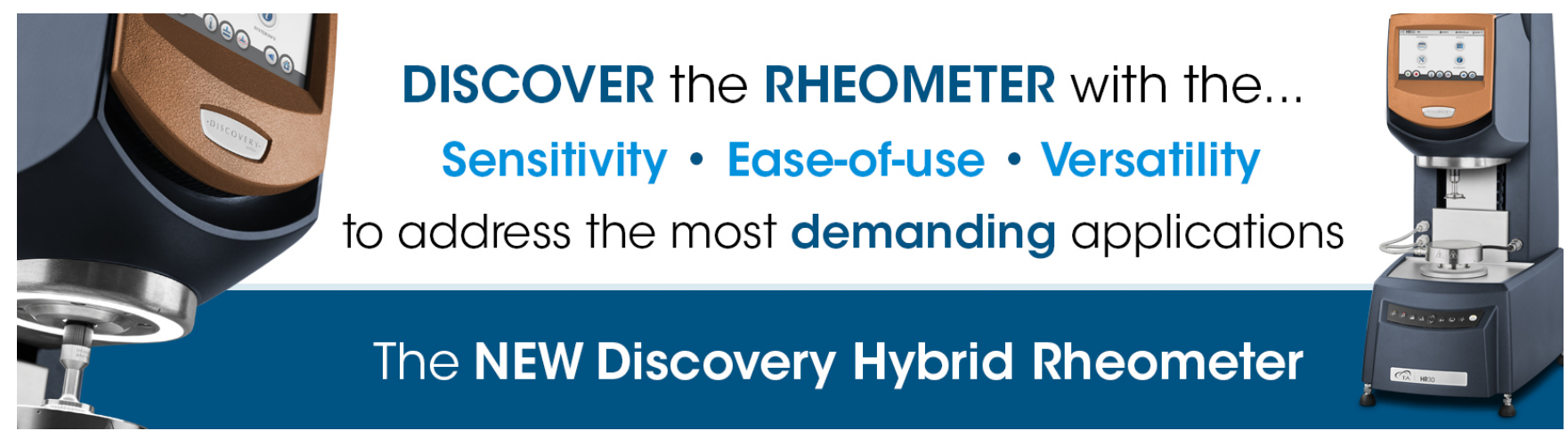




\title{
Evaluating the exit pressure method for measurements of normal stress difference at high shear rates
}

\author{
Dahang Tang, ${ }^{1}$ Flávio H. Marchesini,${ }^{1}$ Ludwig Cardon, ${ }^{1}$ and Dagmar R. D’hooge ${ }^{2,3, a)}$ \\ ${ }^{1}$ Centre for Polymer and Material Technologies, Department of Materials, Textiles and Chemical Engineering, \\ Ghent University, Technologiepark 130, 9052 Zwijnaarde, Belgium \\ ${ }^{2}$ Laboratory for Chemical Technology, Department of Materials, Textiles and Chemical Engineering, Ghent University, \\ Technologiepark 125, 9052 Zwijnaarde, Belgium \\ ${ }^{3}$ Centre for Textile Science and Engineering, Department of Materials, Textiles and Chemical Engineering, \\ Ghent University, Technologiepark 70a, 9052 Zwijnaarde, Belgium
}

(Received 16 January 2020; final revision received 26 March 2020; published 13 April 2020)

\begin{abstract}
A challenge for polymer rheology is the reliable determination of shear dependent first normal stress difference $\left(N_{1}\right.$ values $)$ at high shear rates $\left(>10 \mathrm{~s}^{-1}\right)$. Here, we evaluate the correctness of the commonly applied exit pressure method focusing on polypropylene and high and low density polyethylene melts at $200{ }^{\circ} \mathrm{C}$. It is demonstrated that the linear extrapolation of pressure values toward the die exit, which is a key step in the application of the exit pressure method, is affordable to determine $N_{1}$ values despite that these extrapolated exit pressure values are characterized by a relative deviation of $25 \%-40 \%$. The validity of the exit pressure method is further supported by an excellent match with rheological data from the Laun rule (exponent close to 0.7 ) and a representative simulation of extrudate swelling data in the width and height direction, considering tuned parameters for the Phan-Thien-Tanner constitutive model. Also, the absence of a significant viscous heating effect near the die exit is highlighted based on numerical analysis. (C) 2020 The Society of Rheology. https://doi.org/10.1122/1.5145255
\end{abstract}

\section{INTRODUCTION}

A good understanding of the viscoelastic properties of polymer melts is of great importance to design extrusion and injection molding processes [1-4]. Three shear rate $(\dot{\gamma})$ dependent material functions are essential: (i) the shear/dynamic viscosity $\eta$, (ii) the first normal stress coefficient $\Psi_{1}$, and (iii) the second normal stress coefficient $\Psi_{2}$ [5], which requires the reliable quantification of the shear stress $\left(\sigma_{x y}\right)$, the first normal stress difference $N_{1}\left(\sigma_{x x}-\sigma_{y y}\right)$, and the second normal stress difference $N_{2}\left(\sigma_{y y}-\sigma_{z z}\right)$ as a function of $\dot{\gamma}$ [6]. The associated rheological measurements can be challenging as the conventional rotational rheometers are characterized by an effective shear rate upper limit. The latter rarely surpasses $10^{3} \mathrm{~s}^{-1}$ for polymer solutions and $10 \mathrm{~s}^{-1}$ for polymer melts due to melt edge fracture $[7,8]$, although a cone-partitioned plate rheometer facilitates access to higher $\dot{\gamma}$ [9]. In any case, for normal stress measurements, high accuracy at high $\dot{\gamma}$ still remains a vital issue. In the present work, focus is on the reliable determination of $N_{1}$ values.

To measure $N_{1}$ most commonly, a continuous inline exit pressure method is considered [10], in which focus is on the pressure value at the end of the die, as shown in Fig. 1. This value cannot be directly measured as it is impossible to mount a pressure transducer exactly at the die exit. Instead, extrapolations of the measured pressure profiles are needed along the axial flow and thus the $x$ direction [3,11]. Several

\footnotetext{
a) Author to whom correspondence should be addressed; electronic mail: dagmar.dhooge@ugent.be
}

disturbing factors have been reported [3,7,8,11-19]. As the exit pressure is much smaller than the pressure readings in the die $[8,10-12,19]$, small pressure fluctuations because of a viscous heating effect, pressure-dependent viscosities, and sensitivities of the pressure transducers might render a significant influence on the exit pressure. Also, it is not clear if deviations from the fully developed flow due to velocity rearrangements in vicinity of the die exit matter or not [7,14-18,20,21].

In the present work, a combined experimental and threedimensional (3D) flow modeling study is presented to better quantify the relation of $\dot{\gamma}$ and the success of the exit pressure method, considering polypropylene (PP), low density polyethylene (LDPE), high density polyethylene (HDPE), and PP composite with $20 \%$ graphite fillers (PP/20GP) as melting materials. 3D numerical modeling, as only performed more recently, allows a detailed insight into the flow behavior, especially close to the die exit [22-25]. 3D experimental and simulated data on extrudate swelling are thus additional responses to study the relation of $\dot{\gamma}$ and normal stress differences, therefore opening a new pathway for validation of experimental techniques.

In a first step, we compare the $N_{1}$ values as obtained by the exit pressure method with those following from conventional rotational rheometric analysis and the empirical Laun rule [26]. In a second step, the 3D numerical simulations for the extrudate swell behavior are validated against experimental observations, with the viscoelastic material parameters tuned based on the literature and $N_{1}$ data. In a third step, the exit pressure values with and without the assumption of fully developed flow down to die exit are numerically compared to 


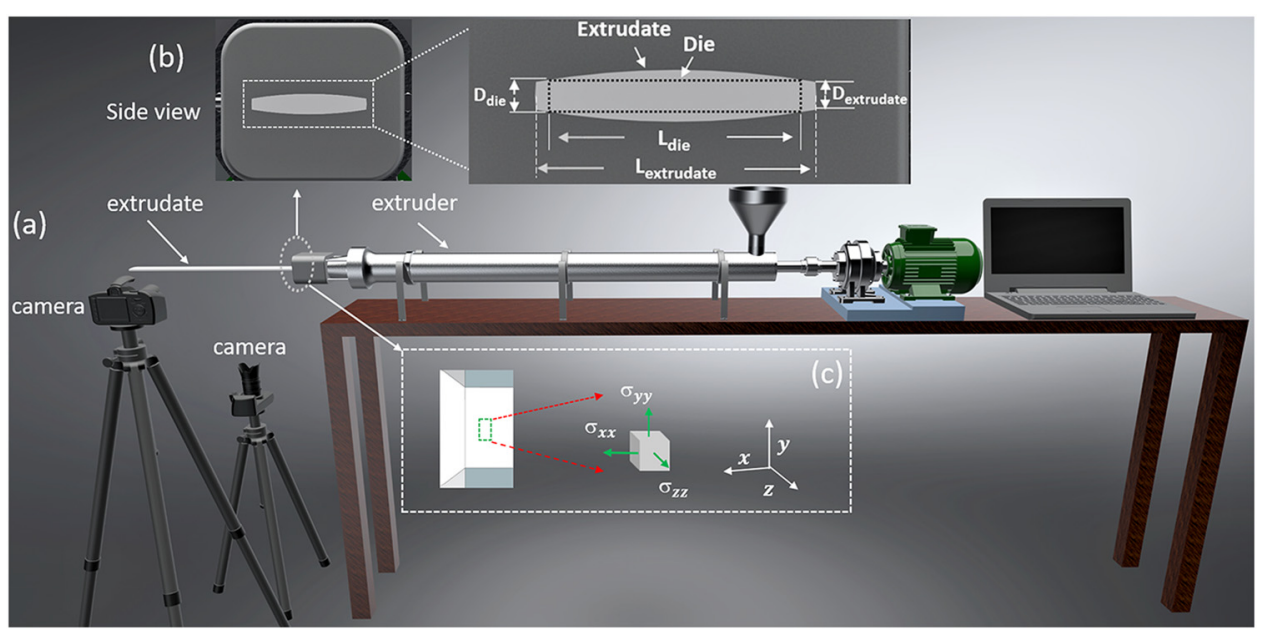

FIG. 1. (a) Principle of extrudate flow from a slit die mounted on an extruder; (b) zoom of the die cross section; (c) the associated normal stresses for an infinitesimally small unit to define the first $\left(N_{1}=\sigma_{x x}-\sigma_{y y}\right)$ and second $\left(N_{2}=\sigma_{y y}-\sigma_{z z}\right)$ normal stress difference.

elucidate the possible contribution of velocity redistributions, thereby the effect on the exit pressure method. In a final step, we numerically focus on the possible impact of a viscous heating effect.

\section{MATERIALS AND EXPERIMENTAL CHARACTERIZATION}

\section{A. Materials}

Four polymer melts, namely, PP (SABIC 575P) melt, LDPE (Lyondellbasell Lupolen 2420H) melt, HDPE (SABIC B5933) melt, and PP/20GP (Asbury 3807), were used with a set temperature of $200{ }^{\circ} \mathrm{C}$. For the pure polymer melts, pellets were directly available. The PP/20GP composite pellets were in contrast made in-house employing twin-screw extrusion with a screw rotational rate of $90 \mathrm{rpm}$ and a processing temperature window of $160-210^{\circ} \mathrm{C}$ using a chopping machine. The polymer melts were all extruded from a single screw extruder (P. Brabender 19 with a screw diameter $D_{s}$ of $19 \mathrm{~mm}, L_{s} / D_{s}$ of $25 ; L_{s}$ : screw length) combined with a slit die \{die length: $220 \mathrm{~mm}$, die height [ $D_{\text {die }}$ in Fig. 1(a)]: $2 \mathrm{~mm}$, and die width $\left[L_{\text {die }}\right.$ in Fig. $\left.1(\mathrm{a})\right]: 20 \mathrm{~mm}$ \} at a screw speed of $10-120 \mathrm{rpm}$ at $200^{\circ} \mathrm{C}$. Important characteristics of the polymer materials are listed in Table I.

\section{B. Mass flow rate and pressure measurements}

The mass flow rates are obtained by collecting extrudates in a given time period. Three pressure transducers were mounted along the die length direction, and the distances

TABLE I. Material characteristics.

\begin{tabular}{lccc}
\hline \hline Material & $\begin{array}{c}\text { Density } \\
\left(\mathrm{g} \mathrm{cm}^{-3} ; 25^{\circ} \mathrm{C}\right)\end{array}$ & $\begin{array}{c}\text { Zero-shear rate viscosity } \\
\left(\mathrm{Pa} \mathrm{s} ; 200{ }^{\circ} \mathrm{C}\right)\end{array}$ & Power law index \\
\hline LDPE & 0.924 & $8.49 \times 10^{3}$ & 0.42 \\
HDPE & 0.959 & $5.12 \times 10^{4}$ & 0.43 \\
PP & 0.905 & $2.24 \times 10^{3}$ & 0.60 \\
PP/20GP & 0.965 & $4.12 \times 10^{4}$ & 0.42 \\
\hline \hline
\end{tabular}

from the die exit are $20 \mathrm{~mm}$ (measurement of $P_{1}$ value), $120 \mathrm{~mm}$ (measurement of $P_{2}$ value), and $170 \mathrm{~mm}$ (measurement of $P_{3}$ value). First, a calibration was conducted for each transducer.

\section{Conventional rheological measurements}

An ARES (TA, Discovery HR-3, US) rheometer was used to perform the temperature-dependent rheological measurements of polymer melts at 180,200 , and $230^{\circ} \mathrm{C}$. The dynamic small amplitude oscillatory measurements were performed with a frequency sweep in an angular velocity $(\omega)$ range of $0.1-500 \mathrm{rad} \mathrm{s}^{-1}$. The steady shear mode tests were carried out by the parallel plate geometry within a small shear rate range of $0.01-5 \mathrm{~s}^{-1}$ to avoid melt edge fracture. The data were corrected for nonlinearity of the shear rate dependent on the radius. Disk samples with a diameter of $25 \mathrm{~mm}$ and a thickness of $2 \mathrm{~mm}$ were made using compression.

To enable an evaluation of conventional analysis tools for normal stress differences, focus has been also on the determination of $N_{1}-N_{2}$ by a parallel plate geometry mode [27,28]. For polymers, generally it holds that $N_{1}>0$ but $N_{2}<0$, and $\left|N_{1}\right| \approx 10\left|N_{2}\right|[27,29]$. The $\left|N_{2}\right| /\left|N_{1}\right|$ value for both HDPE and PP is claimed to be $0.1\left(200^{\circ} \mathrm{C}\right)$ [30,31], which is consistent with our recent simulation results [22]. On the other hand, a value of 0.2 has been put forward for LDPE melt as this polymer is characterized by branched chains [29]. For composites, it has been pointed out that $\left|N_{2}\right| /\left|N_{1}\right|$ increases further [32,33]. Consequently, the ratio is assumed to be 0.2. It should be stressed that these ratios are ball-park values and are fitting parameters in practice, implying the less fundamental nature of this specific determination method.

For comparison, the empirical Laun rule [26] is also utilized to assess $N_{1}$ through relating the dynamic moduli $G^{\prime}$ (storage modulus) and $G^{\prime \prime}$ (loss modulus) for simple steady shear flow in the conventional rheometer,

$$
N_{1}(\dot{\gamma}=\omega)=2 G^{\prime}\left[1+\left(\frac{G^{\prime}(\omega)}{G^{\prime \prime}(\omega)}\right)^{2}\right]^{a} .
$$


Originally, the exponent $\alpha$ was given a value of 0.7 , whereas later on, various works reported values mainly ranging from 0 to $0.7[3,27,34,35]$. In this work, for simplification and without loss of generality, we only adopt $\alpha=0$ and 0.7 , corresponding to a minimum and maximum $N_{1}$ through the Laun rule.

\section{Slit die viscosity measurements}

The shear viscosity $\eta$ for a slit die under ideal laminar and isothermal conditions is given by the ratio of the shear stress at the wall $\left(\sigma_{w}\right)$ and the shear rate at the wall $\left(\dot{\gamma}_{w}\right)$. Considering the Rabinowitch correction it follows that [19]

$$
\begin{gathered}
\sigma_{w}=\frac{H}{(1+e)} * \frac{\partial p}{\partial L}, \\
\dot{\gamma}_{w}=\dot{\gamma}_{a} *\left(\frac{2 n+1}{3 n}\right)=\left(\frac{6 Q}{2 W^{*}(2 H)^{2}}\right)\left(\frac{2 n+1}{3 n}\right),
\end{gathered}
$$

in which $H$ is the half die height, $e$ is the ratio of die height to width, $\partial p / \partial L$ is the pressure gradient along the flow direction, $Q$ is the volumetric flow rate, $W$ is the half die width, $\dot{\gamma}_{a}$ is the apparent shear rate, and $n$ is the power law index of the non-Newtonian melt, which is defined as $n=\mathrm{d} \ln _{w} / \mathrm{d} \ln \dot{\gamma}_{a}$. The $n$ values are given for completeness in Table I. It follows that the $\dot{\gamma}_{w}$ value for the four polymer melts lies in a range of around $10-100 \mathrm{~s}^{-1}$ due to the single extrusion screw speed of 10-120 rpm used in this study.

\section{E. Exit pressure method for measurement of first normal stress difference}

The (conventional) exit pressure theory as used for measuring $N_{1}$ of polymer melt flow through a planar geometry is based on the macroscopic momentum balance theory [36]. More in detail, assuming that a fully developed flow remained until the die exit and that the inertial effect can be neglected due to a low Reynolds (Re) number, Han [14] and Davies et al. [37] derived the following analytic expression linking exit pressure $P_{e x}$ and $N_{1}$ :

$$
N_{1}=P_{\mathrm{ex}}+P_{\mathrm{ex}} \frac{\mathrm{d} \lg P_{\mathrm{ex}}}{\mathrm{d} \lg \sigma_{w}}
$$

Hence, the experimental determination of the relation between $P_{e x}$ and $\sigma_{w}$ is essential.

\section{F. Extrudate swell measurement}

Two high-definition cameras were used to capture the extrudate swell behavior from side and bottom views considering a slit die as depicted in Fig. 1. The details about the measurement method can be found in our previous work focusing solely on the swelling of PP flow [22]. Two swell ratios are considered in the present work, namely, $B_{1}$ and $B_{2}$, corresponding to the swell ratio of the extrudate width and of the extrudate height measured at the edge,

$$
\begin{aligned}
& B_{1}=\frac{L_{- \text {extrudate }}}{L_{- \text {die }}}, \\
& B_{2}=\frac{D_{- \text {extrudate }}}{D_{- \text {die }}}
\end{aligned}
$$

\section{MODELING PRINCIPLES FOR MULTIDIMENSIONAL FLOW CHARACTERISTICS}

\section{A. Details on the governing equations and constitutive model}

In this work, most focus is on isothermal flow conditions, but nonisothermal conditions are also included to evaluate the relevance of viscous heating near the die exit. Assuming incompressible fluids under steady creeping laminar flow conditions without inertial effects and gravity force, the required continuity and momentum equations are given by $[34,38]$

$$
\begin{gathered}
\nabla \cdot \boldsymbol{v}=0, \\
-\nabla p+\nabla \cdot \tau=0, \\
\rho c_{p} v \cdot \nabla T=k \nabla^{2} T+\tau: \nabla v,
\end{gathered}
$$

in which $v$ represents the velocity vector, $p$ the isotropic pressure, $k$ the thermal conductivity, and $\tau$ the extra stress tensor.

\section{Interpretation of the exit pressure theory}

The pressure value $P$ corresponds to the normal force $T_{y y}$ acting on an unit area surface of flow [20,28],

$$
T_{y y}(x, y)=-p(x, y)+\tau_{y y}(x, y),
$$

in which $p(x, y)$ is the isotropic pressure and $\tau_{y y}(x, y)$ is the extra normal stress. For the fully developed flow, $\left|T_{y y}\right|$ is constant and expected to be much larger than $\left|\tau_{y y}\right|$, so that the contribution of $T_{y y}$ only comes from $p(x, y)$. However, with flow disturbance near the die exit, it is no longer reasonable to ignore the contribution of $\tau_{y y}$ to the normal stress $T_{y y}$ or the exit pressure [20]. Under such conditions, the exit pressure $P_{e x}$ needs to be updated,

$$
-P_{\mathrm{ex}}=T_{y y}=-\frac{1}{H} \int_{0}^{H} N_{1} \mathrm{~d} y-\frac{1}{H} \int_{0}^{H} y \frac{\partial \tau_{x y}}{\partial x} \mathrm{~d} y .
$$

The second right term reveals the influence induced by the flow disturbance [3]. The detailed analysis can be found in the work by Boger et al. [7]. Differentiating with respect to $\sigma_{w}$, Eq. (11) can be rewritten as

$$
\begin{aligned}
N_{1}= & P_{\mathrm{ex}}+\tau_{w} \frac{\partial P_{\mathrm{ex}}}{\partial \tau_{w}} \\
& +\frac{\partial}{\partial \tau_{w}} \tau_{w}^{2} \frac{S}{H}\left(1-\int_{0}^{1} f\left(\theta, \tau_{w}, R e\right) \mathrm{d} \theta\right),
\end{aligned}
$$


in which $S$ is the length of the flow disturbance region and $\theta=x / S(0 \leq x \leq S)$. The determination of the integral term $\int_{0}^{1} f\left(\theta, \tau_{w}, R e\right) \mathrm{d} \theta$ is challenging, but in the absence of a velocity rearrangement effect, $f$ is equal to 1 [14], so that Eq. (12) reduces to Eq. (4).

Upon using detailed (3D) simulations, one indirectly accounts for this functional form $f$. Hence, 3D simulations allow us to assess if the pressure variation differs from the linear ideal one.

\section{PTT constitutive model to describe the viscoelastic behavior}

The above system of conservation equations is not closed for non-Newtonian fluids due to the shear dependent extra stress $\tau$ [39], which is for a viscoelastic constitutive model split into a purely viscous part, $\tau_{N}$, and a viscoelastic part, $\tau_{p}$. $\tau_{N}$ is solved by the generalized Newtonian flow model, and $\tau_{p}$ is solved by the basic viscoelastic differential (or integrated) constitutive model $[40,41]$. Note that $\tau_{p}$ is calculated as a sum of $N$ individual $\tau_{p i}$ contributions,

$$
\tau_{p}=\sum_{i=1}^{N} \tau_{p i}
$$

with each individual contribution $\tau_{p i}$ revealed by the differential Phan-Thien-Tanner (PTT) constitutive equation [22],

$$
\exp \left[\frac{\varepsilon_{i} \lambda_{i}}{\eta_{i}} \operatorname{tr}\left(\tau_{p i}\right)\right] \tau_{p i}+\lambda_{i}\left[\left(1-\frac{\xi_{i}}{2}\right) \widetilde{\tau_{p i}}+\frac{\xi_{i}}{2} \widehat{\tau_{p i}}\right]=2 \eta_{i} \mathbf{D}
$$

in which $\lambda_{i}$ and $\eta_{i}$ are the $i$ th $(i=1, \ldots, N)$ relaxation time and viscosity, representing the linear rheological properties of melts. The nonlinear $\xi_{i}$ and $\varepsilon_{i}$ parameters mainly determine the shear and extensional behavior of melts, and $\mathbf{D}$ is the deformation rate tensor.

\section{Additional input for nonisothermal modeling}

For the computations considering the viscous heating effect, nonisothermal flow simulations were carried out and compared to isothermal results $\left(200^{\circ} \mathrm{C}\right)$, applying the conservation equations of mass, momentum, and energy [42]. The temperature-dependent viscosity $\eta$ is obtained with the Arrhenius temperature-shifting function $[42,43]$,

$$
\eta=\sum_{i=1}^{N} \eta_{i} \exp \left[\frac{E}{R_{g}}\left(\frac{1}{T-T_{a}}-\frac{1}{T_{r}-T_{a}}\right)\right]
$$

with the reference temperature $T_{r}$ equal to $200{ }^{\circ} \mathrm{C}$ and $T_{a}$ the absolute zero temperature being $273.15^{\circ} \mathrm{C}, E$ is the activity energy, and $R_{g}$ is the universal gas constant. In addition, focus has been on the calculation of the Nahme number,

$$
N a=\frac{E U^{2}}{k R_{g} T_{r}^{2}}
$$

in which $\eta$ is the nominal viscosity and $U$ is the average velocity in the die. For very low $N a$ numbers, one expects a negligible impact of temperature variations.

\section{B. Geometry and boundary conditions}

In agreement with previous work [25], the 3D flow domain is given in Fig. S1 [59] with one quarter of the flow channel considered for all simulations to reduce the computational cost due to the symmetry planes. Briefly, at the inlet, a fully developed flow with a certain flow rate is considered. At the die walls, a nonslip condition for the velocity field is prescribed. A free surface flow developed outside the die exit is unknown a priori.

For the finite element mesh arrangement, we selected a mesh/grid pattern that progressively becomes more refined upon approaching the singularity at die exit. The grid consists of 12000 elements and 14364 nodes. The refined mesh pattern is shown in Fig. S2 in the supplementary material [59], in which we create denser grids near the die exit and wall: 17000 elements and 19868 nodes. Figure S3 in the supplementary material [59] shows the analysis of the mesh independence on two types of extrudate swell ratios $\left[B_{1}\right.$ and $B_{2}$; Eqs. (5) and (6)] of HDPE melt at $200{ }^{\circ} \mathrm{C}$ at various shear rates. The less refined mesh is utilized for reducing the computational cost.

\section{Numerical solution method}

To solve the system of equations above, a flow solver (POLYFLOW version, ANSYS version 18) is used based on the finite element algorithm. We apply the interpolation functions on the velocity and pressure quadratic and linear, respectively. A discrete elastic viscous stress splitting combined with the streamline-upwind scheme is applied to improve the calculation stability since a geometric singularity exists near the die exit, where a sharp change for the flow boundary condition occurs. In addition, an evolutionary scheme is utilized on the volumetric flow rate in agreement with our previous work [22,25].

\section{RESULTS AND DISCUSSION}

\section{A. Measurement of die pressure profile and shear dependent viscosities}

Figure 2 shows the linearly fitted axial pressure profiles (solid lines) for the four polymer melts at $200{ }^{\circ} \mathrm{C}$ obtained based on the pressure transducer measurements at three positions $\left(P_{1}, P_{2}\right.$, and $\left.P_{3}\right)$ for five wall shear rates. On an overall basis, a good fit is obtained with a coefficient of determination $R^{2}$ very close to 1 in each case (Table $S 1$ in the supplementary material [59]), highlighting the correctness of the experimental setup. The corresponding wall shear stresses are calculated through the slopes of the linear pressure profiles using Eq. (2).

A closer inspection of the experimental data in Fig. 2 reveals that the HDPE melt is characterized by the highest pressure values and changes. Hence, this polymer is most suited to study more in detail if locally pressure drops are altering. It can be deduced that the experimental pressure gradient for HDPE melt is becoming slightly larger toward the die exit. For 

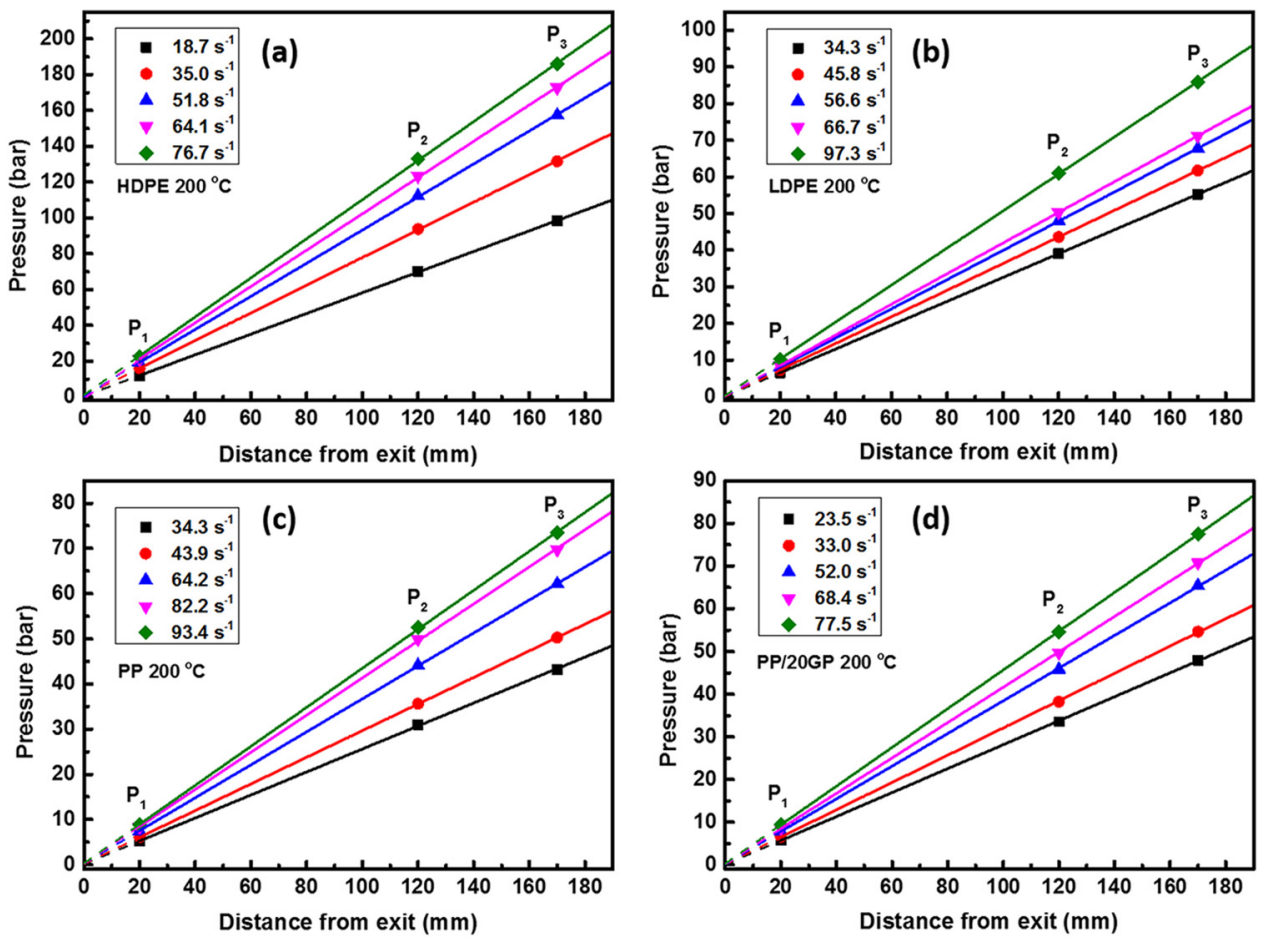

FIG. 2. Lines: Fitted linear axial pressure profiles based on pressure transducer measurements (symbols) for (a) PP, (b) LDPE, (c) HDPE, and (d) PP/20GF polymer melts (material characteristics in Table I) at $200^{\circ} \mathrm{C}$ at different wall shear rates (colors); the raw data and coefficients of determination are given in Table S1 in the supplementary material [59]; extrapolations to die exit ( $x=0$; dashed lines) lead to exit pressure values for the exit pressure method application in Fig. 4; average error bar: $<0.7$ bar.

example, an experimental deviation of $4 \%$ is observed at a shear rate of $76.7 \mathrm{~s}^{-1}$ as $\left(P_{3}-P_{2}\right) / \Delta x=1.06 \mathrm{bar} \mathrm{mm}^{-1}$,

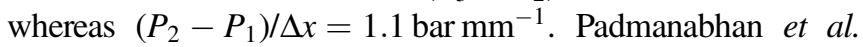
[11] reported that if the pressure is less than $350 \mathrm{bar}$, the effect of pressure on viscosity can be neglected. As pressure calibrations have been performed before the actual measurements, the slightly larger pressure gradient for HDPE upon approaching the die exit might be attributed to a slightly decreasing viscosity from the viscous heating effect or limited polymer degradations.

Figure 3 compares complex $\left(\eta^{*}\right)$ and real $(\eta)$ viscosity values of the dynamic and steady shear test modes for the polymer melts at shear rates up to $500 \mathrm{~s}^{-1}$, using rotational and slit rheometer analysis. A good agreement is observed between the shear (open symbols) and complex (filled symbols) viscosity data for neat PP (red), LDPE (blue), and HDPE (green) at low and high shear rate ranges, which indicates the validity of the Cox-Merz rule for these three melts [44]: $\eta^{*}(\omega)=(\dot{\gamma})$ In contrast, for the PP/20GP composite, the shear viscosity data are much lower than the complex viscosity data, indicating the failure of the Cox-Merz rule for this more complex material. These results are consistent with previous reports [11,45-49] and a possible reason is the deconstruction of the filler-matrix and filler-filler interactions by large strains applied on the flow melt in the nonlinear viscoelastic region [48,50]. As explained in Fig. S4 in the supplementary material [59], the yield stress contribution is only relevant at higher filler loadings (e.g., $40 \mathrm{~m} . \%$ ) and at lower
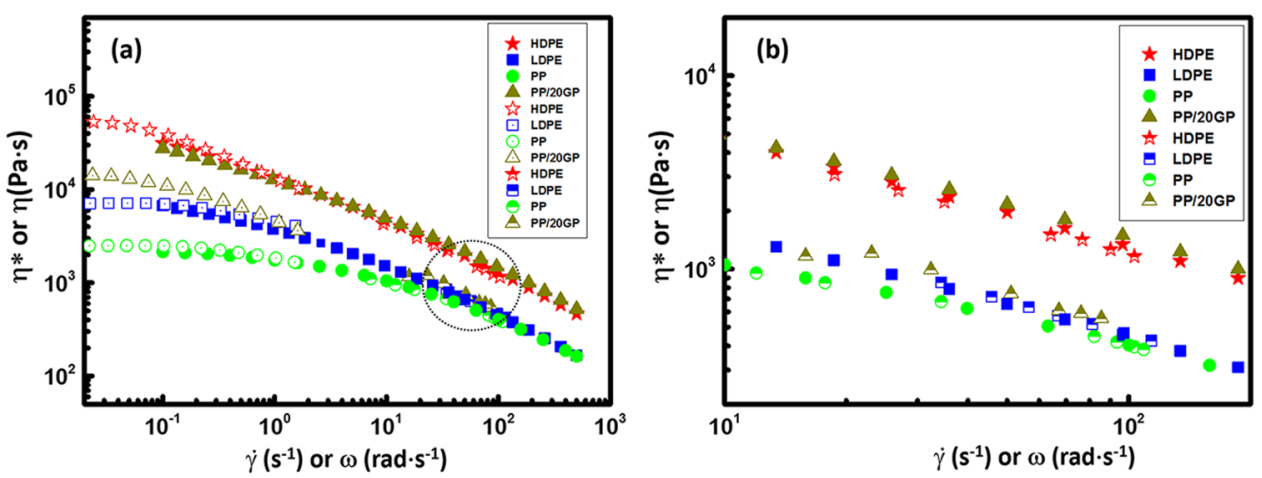

FIG. 3. (a) A comparison between viscosity variations of HDPE (red pentagram), LDPE (blue square), PP (green circle), and PP/20GP (brown triangle) melts at $200^{\circ} \mathrm{C}$ (material characteristics in Table I): complex viscosity $\eta^{*}$ from rotational rheometer: filled symbols; shear viscosity $\eta$ also from rotational rheometer: open symbols; shear viscosity from inline slit die method: half-filled symbols; (b) zoom for indicated region (circle) in Fig. 3(a) at larger shear rates/frequencies; except for the composite, the Cox-Merz rule is obeyed by all polymers. Average error: $<40 \mathrm{~Pa}$ s. 
filler loading (e.g., $10 \mathrm{~m} . \%$ ) the Cox-Merz rule still holds. Overall, the high $R^{2}$ values for Fig. 2 and the applicability of the Cox-Merz rule for PP, HDPE, and LDPE melts indicate that there is no major flaw during the pressure measurements for the neat polymers.

\section{B. Determination of $N_{1}$ via the exit pressure method and through conventional rheological analysis}

Figure 4 shows the extrapolated exit pressure data (open blue symbols) based on the die pressure data in Fig. 2 against the wall shear stresses [Eq. (2)] in a double logarithmic plot, with the corresponding linear fits and associated equations also provided. The $R^{2}$ values are high in any case and thus also for the composite. As in the limit of vanishing wall stress exit pressure values very close to 0 are obtained, one can thus conclude at least at this stage that sufficiently reliable exit pressure data have been retrieved. The slopes in Fig. 4 represent $\mathrm{d} \lg P_{\mathrm{ex}} / \mathrm{d} \lg _{w}$, so that Eq. (4) can be subsequently applied at higher shear rates.

Figure 5 manifests besides these $N_{1}$ values as obtained by the slit rheometer at higher shear rates [exit pressure method; label "E"; Eq. (4); Open blue square symbols] and the $N_{1}$ values based on the Laun rule [Eq. (1); label "L"] as well. Here, a differentiation is made between a red lower and a green higher curve, respectively, corresponding to an exponent $\alpha$ of 0 and 0.7 . In the same figure, conventionally assessed $N_{1}$ values from the rotational rheometer (label "R"; details in the Subsection II C; full filled black symbols) as recorded at lower shear rate are also included.
It is observed in all subplots of Fig. 5 that the difference between the $N_{1-L}$ data for the case of $\alpha=0$ (red lower curves) and 0.7 (green higher curves) becomes larger with the increasing shear rate. As shown in Figs. 5(a)-5(c), for HDPE, LDPE, and PP melts, except for the LDPE melt at the low shear rate range, $N_{1-R}$ variations are qualitatively similar as $N_{1-L}$ variations. This indicates the applicability of the empirical Laun rule for neat polymers, also bearing in mind the good match with $N_{1-E}$ variations at higher shear rates. An optimal exponent toward 0.7 is expected since the green curves are closer to the experimental data than the red curves, which agrees with Baird's work that $N_{1-E}$ values are larger than $N_{1-L}$ data with $\alpha=0$ [3]. Hence, the exit pressure method seems indeed appropriate in measuring $N_{1}$ data for neat polymers, with at least the right magnitude order for neat PP, HDPE, and LDPE melts at high shear rates. Moreover, the slight mismatch for LDPE at the lower shear rates might be attributed to the reason that only a ball-park value for $\left|N_{2}\right| /\left|N_{1}\right|$ has been utilized for the measured $N_{1-L}$ data, as explained above.

It further follows from Fig. 5(d) that the Laun rule [Eq. (1)] fails for the PP/20GP melt, as the $N_{1-L}$ data are significantly larger than the $N_{1-R}$ data, even upon employing an $\alpha$ value of 0 and taking into account that the $N_{1-E}$ values appear to lie in the extrapolation region of $N_{1-R}$ data in different magnitude orders of shear rates. This observation is in line with the work of Winter [51] who suggested that the Laun rule might apply to the Cox-Merz rule obeying materials only. As demonstrated in Fig. 3(b), the Cox-Merz rule is invalid for the composite material. Hence, based on the results in Figs. 2-5, an acceptable consistency is obtained for the neat polymers, and for the composite, it can only be
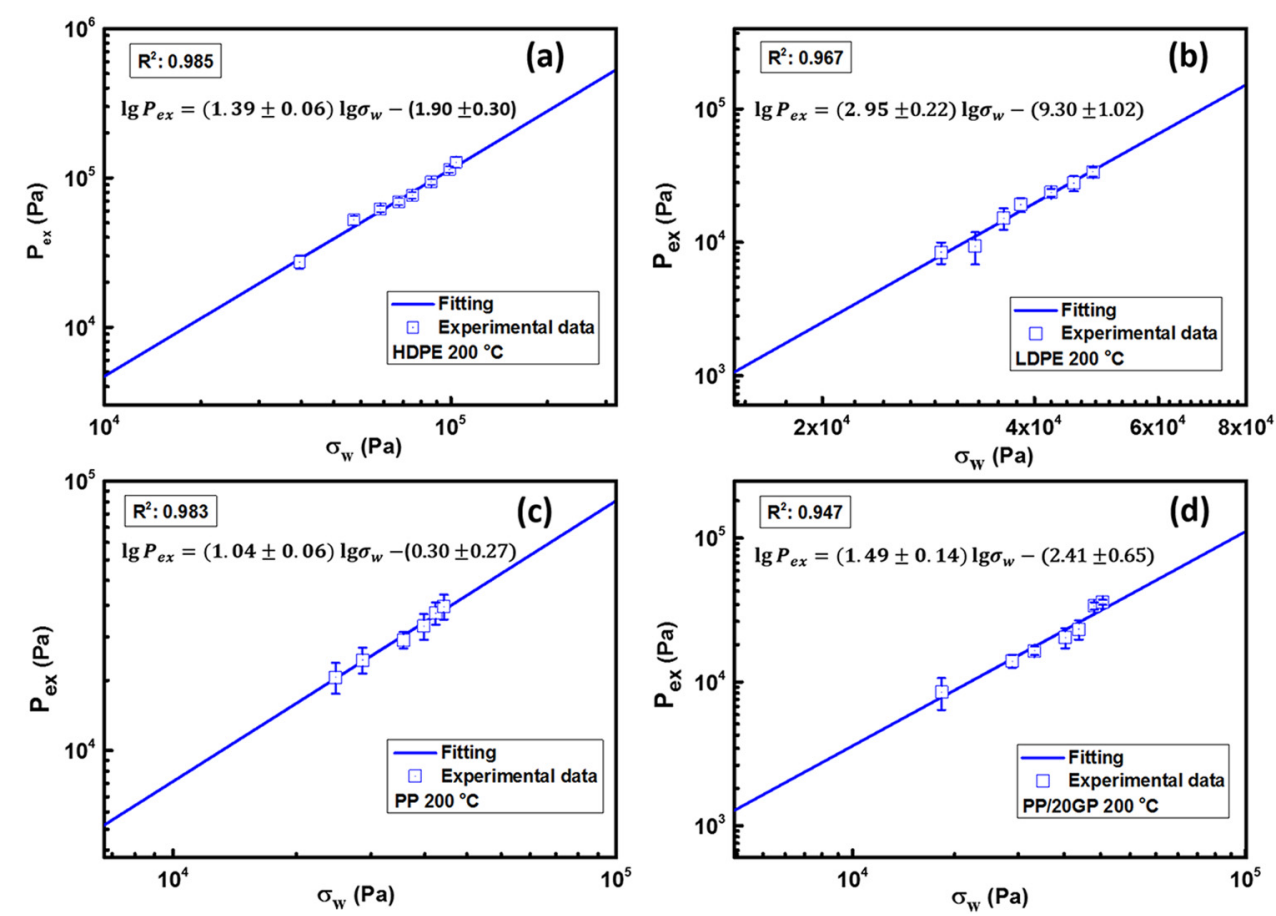

FIG. 4. Exit pressure values (as extrapolated from Fig. 2; open blue symbols; so-called experimental $P_{e x}$ values) versus the wall shear stresses $\sigma_{w}$ [from Eq. (2)] for (a) HDPE, (b) LDPE, (c) PP, and (d) PP/20GP melt at $200^{\circ} \mathrm{C}$. Upon using Eq. (4), the $N_{1}$ values can be obtained as included in Fig. 5 (open blue square symbols). Also given are the linear fit and its equation. The constant term is small so that in the limit of very small shear wall stresses, very small $P_{e x}$ values are obtained. Data from the composite are included for completeness despite that the Cox-Merz rule is not obeyed as indicated in Fig. 3(b). 

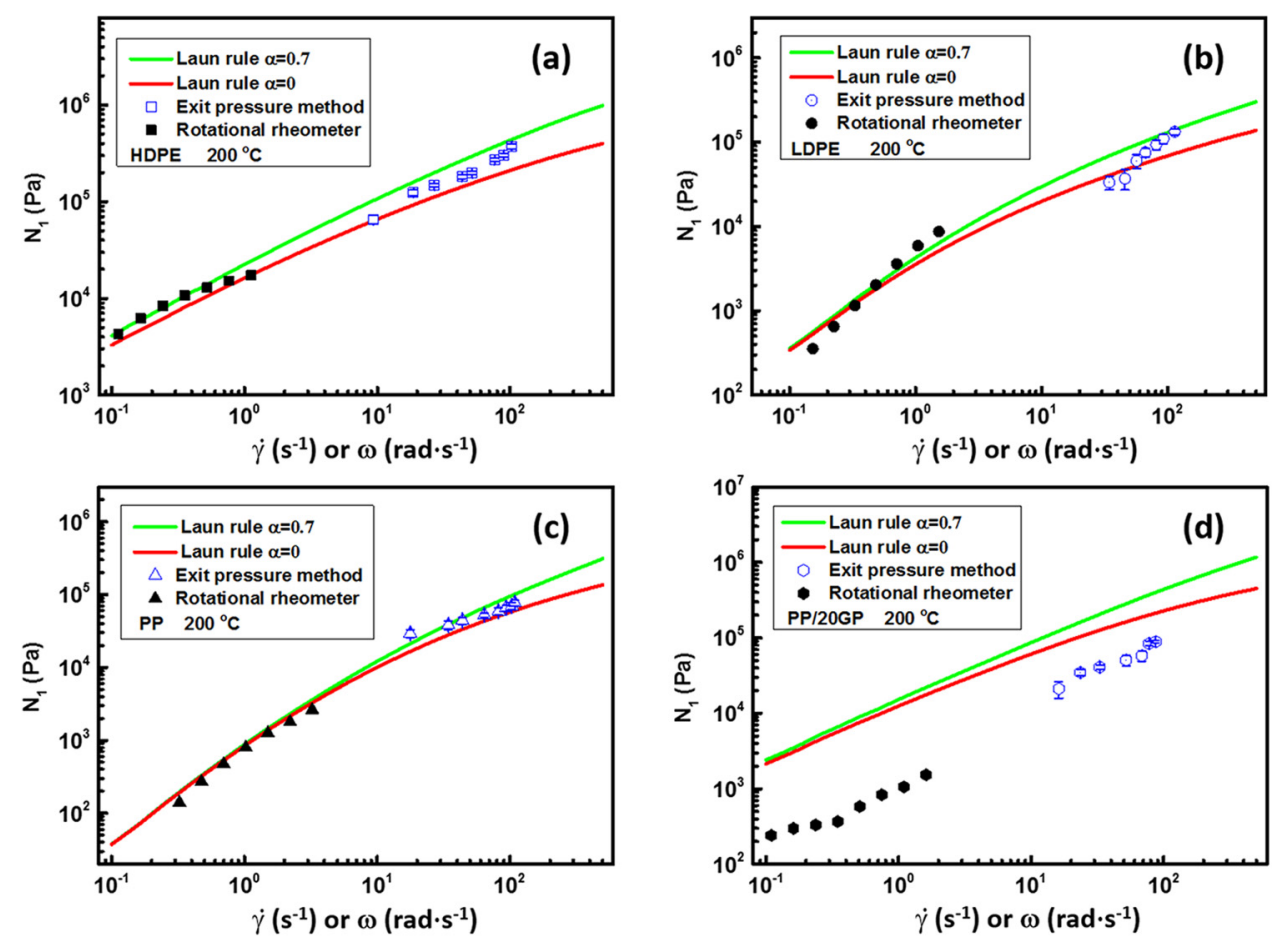

FIG. 5. First normal stress difference $N_{1}$ for polymer melts at various shear rates or sweep frequencies obtained through different methods: (a) LDPE, (b) HDPE, (c) PP, and (d) PP/20GP melts. Curves, black solid symbols and blue open symbols represent, respectively, the $N_{1-L}$ data from the Laun rule [Eq. (1) with exponent 0 (red lower curve) and 0.7 (green higher curve)], the rotational rheometer $\left(N_{1-R}\right.$; Subsection II C), and the exit pressure method [slit rheometer; $N_{1-E}$; Eq. (4)]. For the neat melts, the Laun rule can be applied to a first approximation (exponent close to 0.7). For all melts, the $N_{1-E}$ and $N_{1-R}$ data are consistent.

postulated that the exit pressure method has potential, consistent with a previous report [15].

\section{Numerical analysis to evaluate the assumptions of the exit pressure method}

It is clear from Fig. 5 that a good match of $N_{1}$ data via the exit pressure method [Eq. (4)] and the empirical Laun rule [(exponent $\alpha=0.7)$; Eq. (1)] is obtained for neat polymer melts. To further confirm this interaction, in this modeling section, focus is on the 3D description of experimentally recorded extrudate swell data, which are influenced by normal stress differences [52-54]. Furthermore, the simulations are adopted to study the impact of the velocity distribution and the viscous heating effect.

\section{Determination and validation of PTT input parameters}

In our previous work [22,25], we highlighted the ability of the PTT model to describe 3D extrudate swelling of PP melt at $200{ }^{\circ} \mathrm{C}$ from slit dies. The PTT parameters (Table S2 in the supplementary material [59]) followed from tuning to different types of rheological data. Figure 6(c) (purple filled curve)
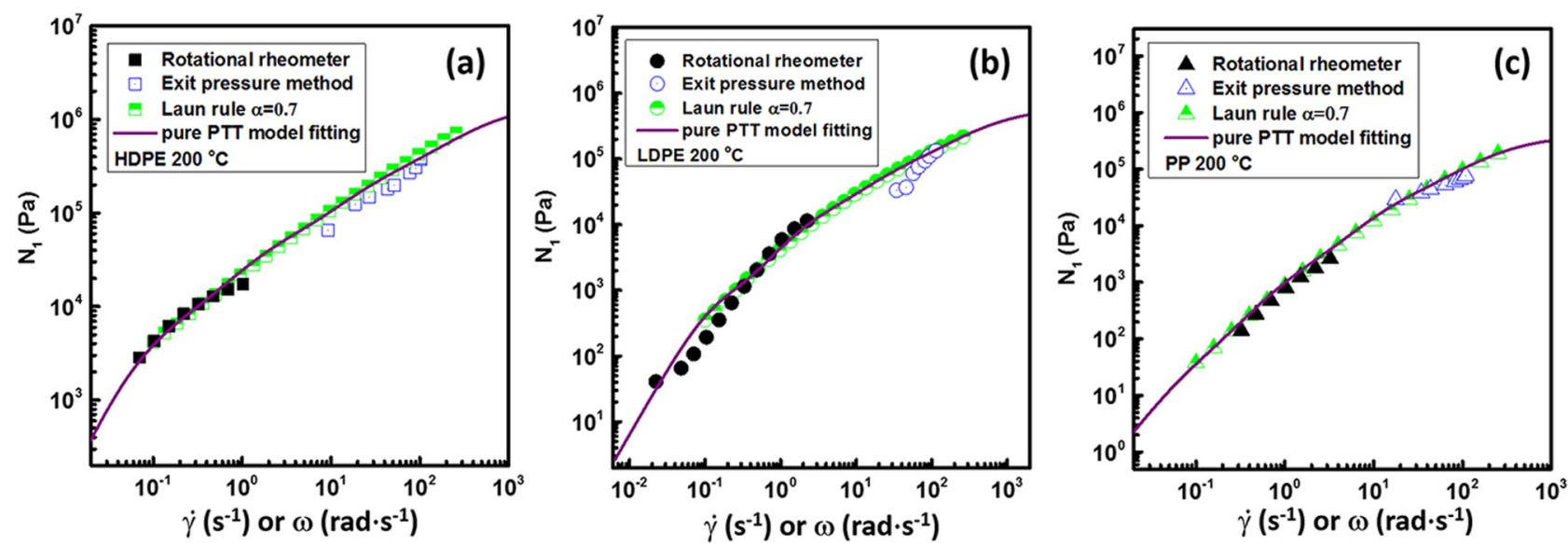

FIG. 6. $N_{1}$ data of HDPE, LDPE, and PP melts at $200^{\circ} \mathrm{C}$. Symbols indicate experimental data from the exit pressure method [Eq. (4); blue open symbols], rotational rheometer (cf. Subsection II C, black filled symbols), and the Laun rule [Eq. (1); green half-filled symbols; exponent of 0.7], while the purple curves correspond to overall fits with the PTT model. The obtained PTT parameters are highlighted in Table II (HDPE and LDPE) and Table S2 (PP) in the supplementary material [59]. It follows that the PTT model curves are consistent with all data and thus also the data as recorded by the exit pressure method. 
TABLE II. PTT parameters for HDPE and LDPE melts at $200^{\circ} \mathrm{C}$; those for PP are given in the supplementary material [59] and taken from the previous work [22].

\begin{tabular}{|c|c|c|c|c|c|c|c|c|c|}
\hline Materials & Mode & $\lambda_{i}(\mathrm{~s})$ & & $\begin{array}{l}\text { TT mode } \\
\eta_{i}(\mathrm{~Pa} \cdot \mathrm{s})\end{array}$ & & $\varepsilon_{i}$ & & $\xi_{i}$ & \\
\hline & 1 & $\lambda_{1}$ & 0.005 & $\eta_{1}$ & 890 & $\varepsilon_{1}$ & 0.18 & $\xi_{1}$ & 0.18 \\
\hline & 2 & $\lambda_{2}$ & 0.072 & $\eta_{2}$ & 3882 & $\varepsilon_{2}$ & 0.23 & $\xi_{2}$ & 0.18 \\
\hline \multirow[t]{3}{*}{ HDPE } & 3 & $\lambda_{3}$ & 1.04 & $\eta_{3}$ & 12977 & $\varepsilon_{3}$ & 0.23 & $\xi_{3}$ & 0.23 \\
\hline & 4 & $\lambda_{4}$ & 15 & $\eta_{4}$ & 33595 & $\varepsilon_{4}$ & 0.25 & $\xi_{4}$ & 0.3 \\
\hline & 1 & $\lambda_{1}$ & 0.005 & $\eta_{1}$ & 325 & $\varepsilon_{1}$ & 0.2 & $\xi_{1}$ & 0.15 \\
\hline \multirow[t]{3}{*}{ LDPE } & 2 & $\lambda_{2}$ & 0.063 & $\eta_{2}$ & 1123 & $\varepsilon_{2}$ & 0.22 & $\xi_{2}$ & 0.16 \\
\hline & 3 & $\lambda_{3}$ & 0.794 & $\eta_{3}$ & 3290 & $\varepsilon_{3}$ & 0.20 & $\xi_{3}$ & 0.22 \\
\hline & 4 & $\lambda_{4}$ & 10 & $\eta_{4}$ & 2950 & $\varepsilon_{4}$ & 0.05 & $\xi_{4}$ & 0.4 \\
\hline
\end{tabular}

shows that the PTT predicted $N_{1}$ values for PP are in agreement with experimentally recorded values with the exit pressure method [Eq. (4)] and a Laun rule curve [Eq. (1)] considering an $\alpha$ value of 0.7 .

This good linkage of the PTT based modeling approach with the exit pressure method as well as the Laun rule curve is further confirmed in Figs. 6(a) and 6(b) for HDPE and LDPE melts still at $200^{\circ} \mathrm{C}$. The good tuning of the rheological parameters for both polymer melts, as conducted in the present work, is presented in Fig. S5 in the supplementary material [59]. The corresponding PTT model parameters for these PE melts are given in Table II.

\section{Comparison between extrudate swell simulations and experimental data}

Figures 7(a) and 7(b) compare the evolution of the simulated extrudate swell behavior of the extrudate width $\left[B_{1}\right.$;
Eq. (5)] for HDPE and LDPE melts with the experimental data at various shear rates. A good agreement is observed in terms of $B_{1}$, which increases with the shear rate and flow distance away from the die exit. This is in agreement with literature general trends [31,55,56]. A significantly smaller increase of $B_{1}$ is noticed as the wall shear rate varies from 35 to $51.8 \mathrm{~s}^{-1}$ compared to the variation from 9.3 to $35 \mathrm{~s}^{-1}$. This is similar to the predicted extrudate swell behavior for HDPE melt by Ansari et al. [34]. Furthermore, $B_{1}$ of the HDPE melt is larger than that of the LDPE melt, indicating the more significant elastic property for HDPE melt at the temperature of $200{ }^{\circ} \mathrm{C}$. This can be specifically postulated as the corresponding shear rate is even lower for the former case.

Figures $7(\mathrm{c})$ and $7(\mathrm{~d})$ show the corresponding swell behavior at $200{ }^{\circ} \mathrm{C}$ of the extrudate height at the edge $\left[B_{2}\right.$; Eq. (6)]. It follows that a "negative" swelling or so-called contraction flow behavior $\left(B_{2}<1\right)$ is observed. This swell behavior highlights the strength of $3 \mathrm{D}$ simulations with a full
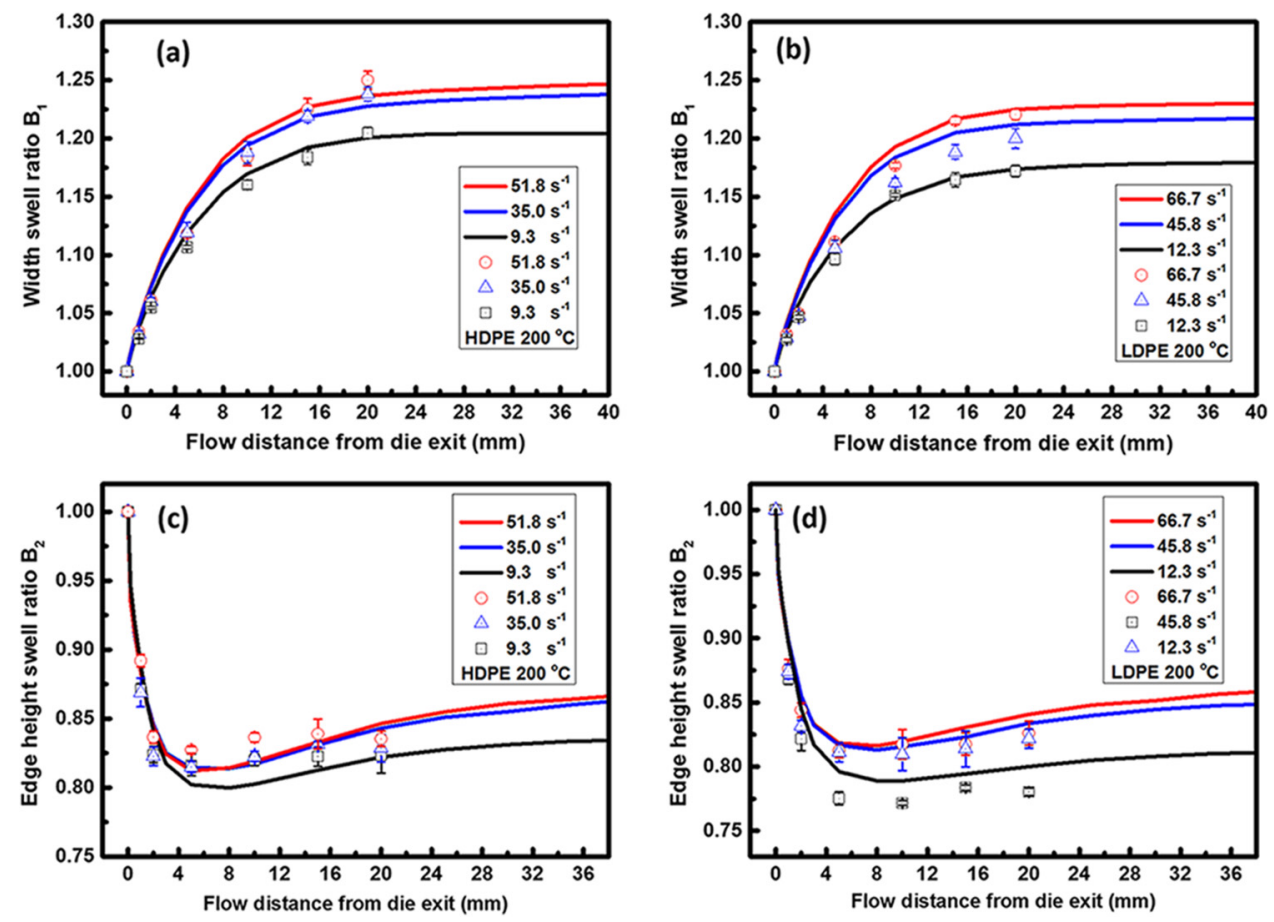

FIG. 7. Simulated (curves; 3D isothermal simulations using the PTT model) and experimental (symbols) evolutions of the swell behavior of the extrudate width $\left[B_{1}\right.$; Eq. (5); (a) and (b)] and of the height at the edge $\left[B_{2}\right.$; Eq. (6); (c) and (d)] for HDPE and LDPE melts at $200{ }^{\circ} \mathrm{C}$ at different shear rates. Similar plots for PP melt are shown in Fig. S6 in the supplementary material [25,59]. Good descriptions are observed, further validating the relevance of Eq. (4). 
preservation of mass and cannot be captured by simplified two-dimensional simulations [25]. A good agreement between the experimental data and simulation results can be noted again for both HDPE and LDPE melts. In the supplementary material (Fig. S6) [59], a similar agreement for the PP melt is included.

Based on the numerical flow results regarding extrudate swell, it can thus be concluded that the $N_{1}$ values as following from the Laun rule (exponent of 0.7 ) and the exit pressure method [Eq. (4)] describe well the elastic properties of PP, HDPE, and LDPE melts at $200^{\circ} \mathrm{C}$. This further supports the application of the conventional exit pressure method, although a further validation can still be conducted with respect to its ideal flow assumptions, as covered below.

\section{Numerical evaluation of impact of flow disturbances near the die exit}

To investigate the effect of flow disturbances near the die exit in Fig. S7 in the supplementary material [59], a numerical comparison is performed between the simulated exit pressure values as obtained by the detailed 3D modeling and those assuming simplified linear extrapolation. It is seen that the deviation between both types of simulations starts at around $1 \mathrm{~mm}$ before the die exit, which is in agreement with the literature stating disturbances at one or two times of the half die height (here $H=1 \mathrm{~mm}$ ) [7,20]. Furthermore, a sharp increase in the simulated pressure value very close to the die exit is noted with the 3D model. This increase is related to a numerical stress singularity near the die exit, as shown in Fig. 8 for the three neat melts and two wall shear rates. Note that for HDPE, the stronger $N_{1}$ increases in Fig. 8 are a consequence of the stronger pressure variations in Fig. 2 and Fig. S7 in the supplementary material [59], and the higher elasticities as discussed in Fig. 7.

The nature of exit singularity for viscoelastic models is still unclear as being a real physical phenomenon or an artificial prediction or a combination of both [57]. It has been

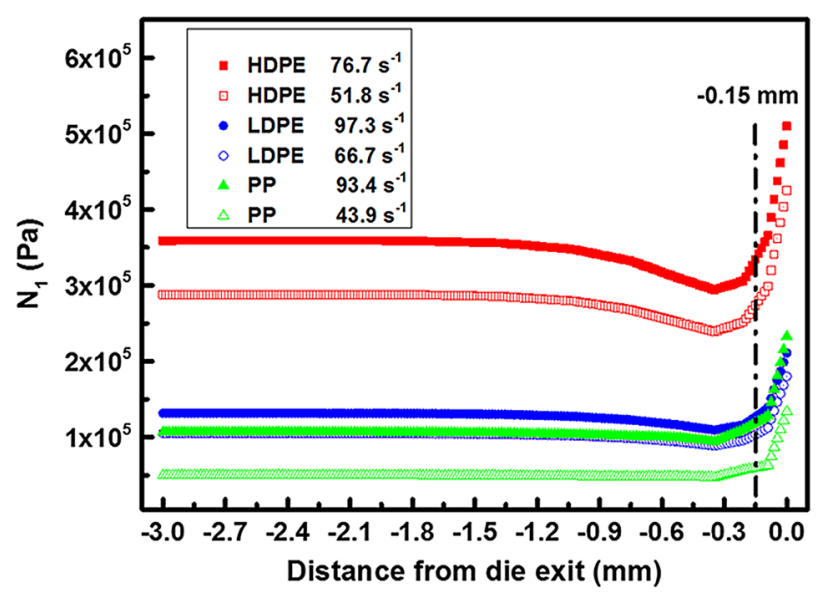

FIG. 8. $N_{1}$ data along the die wall for LDPE and HDPE melts at lower and higher wall shear rates (colors) according to a detailed $3 \mathrm{D}$ model so with velocity redistribution at $200^{\circ} \mathrm{C}$. The dashed line indicates the position at which we calculate the exit pressure value with the $3 \mathrm{D}$ full model considering the velocity redistribution (so-called $P_{e x, r}$ value) to minimize the impact of a possible artificial effect [57]. indicated that mesh refinement enhances the intensity of the stress singularity [22,57] and thereby results in a larger pressure values near the exit. This has been noticed in the present work as well with also a delay for the drastic increase taking place in line with literature data [58]. Hence, it is recommended to consider an intermediate solution and to deliver with a detailed flow modeling at least an underestimated but not an overestimated value of the exit pressure. Therefore, we evaluate the pressure value at the position where the $N_{1}$ value abruptly starts increasing. Figure 8 displays that this position is around at a distance of $0.1-0.2 \mathrm{~mm}$ before the die exit. For uniformity, we fix this position at $0.15 \mathrm{~mm}$ before the die exit for all simulations. For clarity, we denote this modeled exit pressure value as $P_{e x, r}$ as opposed to $P_{e x}$ as based on Eq. (4) and thus extrapolation.

Importantly, this distance of $0.15 \mathrm{~mm}$ is in the extrapolated region in Fig. 2, and thus a natural question is to quantify the deviations between linearly extrapolated and actually calculated values accounting for velocity redistributions. Figure S8 in the supplementary material [59] shows that for all neat melts, the flow disturbance in the vicinity of the die exit leads to a significantly higher pressure value. The relative contribution stemming from the velocity redistribution $R_{v}$, which is defined as $\left(P_{e x, r}-P_{e x}\right) / P_{e x, r}$ amounts to $\sim 25 \%-$ $40 \%$. Increasing the shear rate decreases slightly the value of $R_{v}$, which agrees with the report by Han [20].

These insights at first sight indicate that Eq. (4) is unsuited as there are significant deviations between $P_{e x}$ and $P_{e x, r}$ values. However, it should be reminded that the actual goal is the reliable determination of $N_{1}$. Therefore, Fig. 9 (top) presents the update of Fig. 4 for the two PE melts using simulated $P_{e x}$ values as obtained from linear extrapolation of the 3D modeling results. We start here at a distance sufficiently away from the die exit to ensure the linearity according to the method. Similar slopes as in Figs. 4(a) and 4(b) are observed, indicating a limited effect on the $N_{1}$ determination.

The latter is indeed confirmed in Fig. 9 (bottom) in which the lines from Fig. 9 (top) are used to obtain the corresponding $N_{1}$ values (red filled square symbols) formally according to Eq. (4). It follows that these $N_{1}$ data are consistent with the $N_{1}$ data according to the green Laun rule curve with an exponent $\alpha$ of 0.7 . This highlights the relevance of the exit pressure method as these Laun rule data are in turn consistent with the $N_{1}$ values recorded based on the exit pressure method thus upon applying Eq. (4) with the actual experimental pressure profiles (cf. discussion Fig. 5). In addition, we show in Fig. 9 (bottom) that the $N_{1}$ data for the PE melts as simulated by the detailed 3D model (evaluation at wall), thus employing the PTT model in the POLYFLOw software while considering velocity redistributions (purple open square symbols), also agree with the Laun rule data. Hence, the assumption of fully developed flow down to the die exit is valid for the $N_{1}$ calculation by means of the exit pressure method.

\section{Impact of viscous heating effect}

The numerical analysis mentioned above is performed under isothermal flow conditions. However, the viscous heating effect of the viscoelastic flow might influence the 

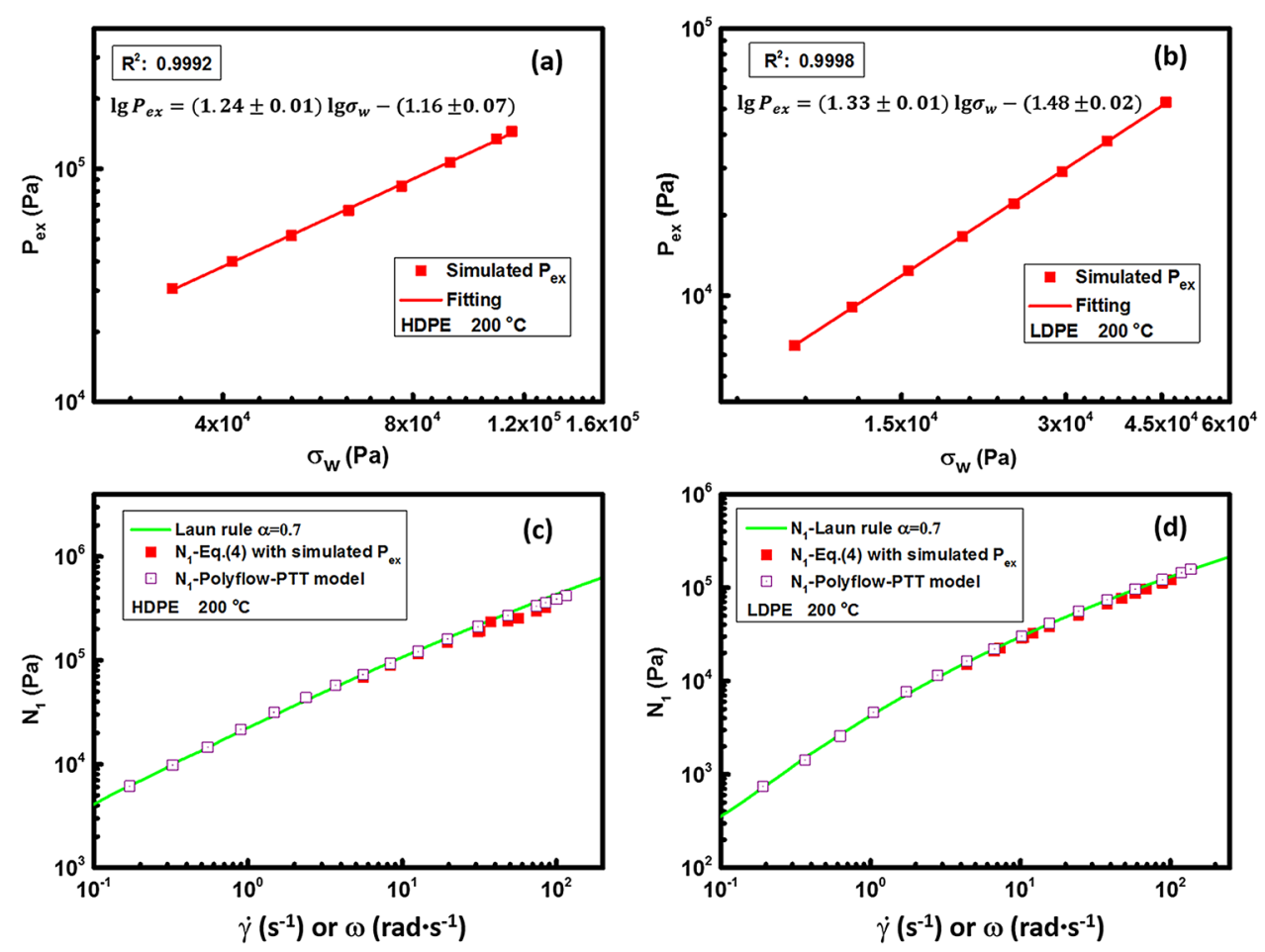

FIG. 9. Top: Simulated exit pressure by linear extrapolation (sufficiently far away from the die exit) as a function of the simulated wall shear stress $\sigma_{w}$ : (a) LDPE and (b) HDPE at $20{ }^{\circ} \mathrm{C}$; full red curve: linear fit. Bottom: comparison of the normal stress difference $N_{1}$ as obtained with the full 3D model thus accounting for velocity distributions (purple open symbols; evaluation at wall), as calculated based on the simulated extrapolated $P_{e x}$ in (a) and (b) with Eq. (4) (red filled square symbols) and as obtained based on the Laun rule with $\alpha=0.7$ (full green cruves; consistent with experimental exit pressure values in Fig. 5).

temperature field inside the flow channel [43], thus affecting the pressure field. The fit of the temperature-dependent rheological properties of polymer melts is shown in Fig. S9 in the supplementary material [59], with the material parameters required presented in Table S3 in the supplementary material [59] for the three polymer melts.

As explained in Fig. S10 in the supplementary material [59], flow simulations indicate a negligible effect of temperature gradients, implying a very low $N a$ number. Indeed, for HDPE, LDPE, and PP melts, the $N a$ number is $0.032,0.035$, and 0.09 at the highest shear rate, indicating a very weak viscous heating effect $[34,43]$. Consequently, the $N_{1}$ measurements by means of the exit pressure method is thought to be free from this effect, at least considering the experimental ranges investigated in this work.

\section{v. CONCLUSIONS}

For neat polymer melts at $200{ }^{\circ} \mathrm{C}$, we have comprehensively evaluated the exit pressure method via the slit rheometer to measure $N_{1}$ values at high shear rates, which cannot be obtained by a conventional rotational rheometer due to the melt edge fracture. In this method, the (quasi-)linear axial pressure profiles for the polymers at various wall shear rates are first extrapolated to the die exit, yielding the corresponding exit pressure values, which in turn are related to the associated shear stresses to deliver $N_{1}$ values.

For these neat polymers, the shear viscosity data measured from the inline slit die method agree very well with those from the rotational rheometer at low shear rates as well as the associated measured complex viscosity data over a wide shear rate range, indicating the validity of the empirical Cox-Merz rule. $N_{1}$ values at the shear rate magnitude order of $10-100 \mathrm{~s}^{-1}$ as measured by the exit pressure method lie additionally in the value range calculated by the Laun rule with an exponent $\alpha$ of 0 and 0.7 .

Extrudate swell behavior, which is related to normal stress differences, has been also utilized to further assess the validity of the exit pressure method for these neat polymer melts. 3D numerical simulations have been performed, using the differential viscoelastic multimode PTT model. The corresponding material parameters have been obtained by tuning the rheological functions including fitting the $N_{1}$ data obtained from the empirical Laun rule that are consistent with the exit pressure method based $N_{1}$ data. A good agreement between the predicted extrudate swell and experimental measurements for the neat polymer melts is observed, which further confirms that the exit pressure method is appropriate to determine $N_{1}$ values.

To address the disagreement in the field on the exit pressure method stemming from the skeptical assumption of fully developed flow down to die exit, we also compared the numerical exit pressure values in terms of accounting for the flow redistributions near the die exit or not doing so. Although the pressure contribution from velocity rearrangement near the die exit accounts for $\sim 25 \%-40 \%$ at various shear rates, this deviation diminishes in impact upon focusing on the actual simulated $N_{1}$ values, which are consistent with those by the exit pressure method. In addition, a negligible effect coming from viscous heating of polymer melts on the 
pressure field near the die exit has been demonstrated. Hence, the exit pressure method can be reliably utilized for the neat polymers studied.

Future work will be directed to also investigate composites. In this respect, the current study has already shown that the Cox-Merz and the Laun rule fail to describe the viscosity data for a PP composite. Even though the Laun rule fails, the $N_{1}$ values obtained with the exit pressure method appear to be in the extrapolation region of those obtained by the rotational rheometer. Hence, the exit pressure method also seems to have potential for more complex materials. Additionally, future work could focus on the evaluation at even higher shear rates.

\section{ACKNOWLEDGMENTS}

D.T. appreciates funding from the China Scholarship Council (Grant No. 201606240114) for the Ph.D. study at Ghent University. The authors thank Tom Van Waeleghem for support in designing Fig. 1. They also thank Dr. Zhongguo Zhao from the College of Polymer Science and Engineering, Sichuan University, P. R. China, for assistance with the rheological measurements.

\section{REFERENCES}

[1] Tian, H., D. Zhao, M. Wang, G. Jin, and Y. Jin, "Study on extrudate swell of polypropylene in double-lumen micro profile extrusion," J. Mater. Process. Tech. 225, 357-368 (2015).

[2] Thiébaud, F., and J. Gelin, "Characterization of rheological behaviors of polypropylene/carbon nanotubes composites and modeling their flow in a twin-screw mixer," Compos. Sci. Technol. 70, 647-656 (2010).

[3] Baird, D. G., "First normal stress difference measurements for polymer melts at high shear rates in a slit-die using hole and exit pressure data," J. Non-Newtonian Fluid Mech. 148, 13-23 (2008).

[4] Robertson, B., R. L. Thompson, T. C. McLeish, and I. Robinson, "Theoretical prediction and experimental measurement of isothermal extrudate swell of monodisperse and bidisperse polystyrenes," J. Rheol. 61, 931-945 (2017).

[5] Jensen, E. A., "Measurements of first and second normal stress differences in a polymer melt," J. Non-Newtonian Fluid Mech. 148, 41-46 (2008).

[6] Bird, R. B., R. C. Armstrong, and O. Hassager, Dynamics of Polymeric Liquids (Wiley, New York, 1987), Vol. 1: Fluid Mech.

[7] Boger, D. V., and M. M. Denn, "Capillary and slit methods of normal stress measurements," J. Non-Newtonian Fluid Mech. 6, 163-185 (1980).

[8] Tuna, N., and B. Finlayson, "Exit pressure experiments for low density polyethylene melts," J. Rheol. 32, 285-308 (1988).

[9] Konaganti, V. K., M. Ansari, E. Mitsoulis, and S. G. Hatzikiriakos, "The effect of damping function on extrudate swell," J. Non-Newtonian Fluid Mech. 236, 73-82 (2016).

[10] Chan, T., B. Pan, and H. Yuan, "An experimental study of exit pressures for polymer melts," Rheol. Acta 29, 60-70 (1990).

[11] Padmanabhan, M., and M. Bhattacharya, "In-line measurement of rheological properties of polymer melts," Rheol. Acta 33, 71-77 (1994).

[12] Lodge, A., and L. De Vargas, "Positive hole pressures and negative exit pressures generated by molten polyethylene flowing through a slit die," Rheol. Acta 22, 151-170 (1983).

[13] Tuna, N. Y., and B. A. Finlayson, "Exit pressure calculations from numerical extrudate swell results," J. Rheol. 28, 79-93 (1984).
[14] Han, C. D., "On silt and capillary die rheometry," Trans. Soc. Rheol. 18, 163-190 (1974).

[15] Han, C. D., "Rheological properties of calcium carbonate-filled polypropylene melts," J. Appl. Polym. Sci. 18, 821-829 (1974).

[16] Han, C. D., and L. H. Drexler, "The stress distribution of polymer melts in the exit region,” Trans. Soc. Rheol. 17, 659-665 (1973).

[17] Carreau, P. J., L. Choplin, and J. R. Clermont, "Exit pressure effects in capillary die data,” Polym. Eng. Sci. 25, 669-676 (1985).

[18] Choplin, L., and P. Carreau, "Excess pressure losses in a slit," J. Non-Newtonian Fluid Mech. 9, 119-146 (1981).

[19] Laun, H. M., "Polymer melt rheology with a slit die," Rheol. Acta 22, 171-185 (1983)

[20] Han, C. D., "What is the role of "pressure" in the use of capillary and slit flows to determine the shear-rate dependent viscosity of a viscoelastic fluid?," Polym. Eng. Sci. 48, 1126-1140 (2008).

[21] Han, C., M. Charles, and W. Philippoff, "Rheological implications of the exit pressure and die swell in steady capillary flow of polymer melts. I. The primary normal stress difference and the effect of L/D ratio on elastic properties," Trans. Soc. Rheol. 14, 393-408 (1970).

[22] Tang, D., F. H. Marchesini, D. R. D'hooge, and L. Cardon, "Isothermal flow of neat polypropylene through a slit die and its die swell: Bridging experiments and 3D numerical simulations," J. Non-Newtonian Fluid Mech. 266, 33-45 (2019).

[23] Schoonen, J. F., F. H. Swartjes, G. W. Peters, F. Baaijens, and H. E. Meijer, "A 3D numerical/experimental study on a stagnation flow of a polyisobutylene solution," J. Non-Newtonian Fluid Mech. 79, 529-561 (1998).

[24] Sirakov, I., A. Ainser, M. Haouche, and J. Guillet, "Three-dimensional numerical simulation of viscoelastic contraction flows using the Pom-Pom differential constitutive model," J. Non-Newtonian Fluid Mech. 126, 163-173 (2005).

[25] Tang, D., F. H. Marchesini, L. Cardon, and D. R. D'hooge, "Three-dimensional flow simulations for polymer extrudate swell out of slit dies from low to high aspect ratios," Phys. Fluids 31, 093103 (2019).

[26] Laun, H., "Prediction of elastic strains of polymer melts in shear and elongation,” J. Rheol. 30, 459-501 (1986).

[27] Kádár, R., I. F. Naue, and M. Wilhelm, "First normal stress difference and in-situ spectral dynamics in a high sensitivity extrusion die for capillary rheometry via the "hole effect"," Polymer 104, 193-203 (2016).

[28] Macosko, C. W., and R. G. Larson, Rheology: Principles, Measurements, and Applications (Wiley, New York, 1994).

[29] Meissner, J., R. Garbella, and J. Hostettler, "Measuring normal stress differences in polymer melt shear flow," J. Rheol. 33, 843-864 (1989).

[30] Mitsoulis, E., H. J. Luger, J. Miethlinger, and W. Friesenbichler, "Flow behavior of a polypropylene melt in capillary dies," Int. Polym. Process. 33, 642-651 (2018).

[31] Konaganti, V., E. Behzadfar, M. Ansari, E. Mitsoulis, and S. Hatzikiriakos, "Extrudate swell of high density polyethylenes in slit (flat) dies," Int. Polym. Process. 31, 262-272 (2016).

[32] Barnes, H. A., "A review of the rheology of filled viscoelastic systems," Rheol. Rev. 2003, 1-36 (2003).

[33] Ohl, N., and W. Gleissle, "The second normal stress difference for pure and highly filled viscoelastic fluids," Rheol. Acta 31, 294-305 (1992).

[34] Ansari, M., E. Mitsoulis, and S. G. Hatzikiriakos, "Capillary extrusion and swell of a HDPE melt exhibiting slip," Adv. Polym. Tech. 32, E369-E385 (2013).

[35] Wen, Y. H., H. C. Lin, C. H. Li, and C. C. Hua, "An experimental appraisal of the Cox-Merz rule and Laun's rule based on bidisperse entangled polystyrene solutions," Polymer 45, 8551-8559 (2004). 
[36] Lodge, A., Elastic Liquids (Academic, New York, 1964).

[37] Davies, J., J. Hutton, and K. Walters, "Theory for normal stresses in slits and capillaries," J. Phys. D Appl. Phys. 6, 2259 (1973).

[38] Mu, Y., L. Hang, A. Chen, G. Zhao, and D. Xu, "Influence of die geometric structure on flow balance in complex hollow plastic profile extrusion,” Int. J. Adv. Manuf. Tech. 91, 1275-1287 (2017).

[39] Gujrati, P. D., and A. I. Leonov, Modeling and Simulation in Polymers (Wiley-VCH, Weinhem, 2010).

[40] Aloku, G. O., and X. F. Yuan, "Numerical simulation of polymer foaming process in extrusion flow," Chem. Eng. Sci. 65, 3749-3761 (2010).

[41] Mitsoulis, E., M. Battisti, A. Neunhäuserer, L. Perko, and W. Friesenbichler, "Flow behavior of PP-polymer nanocomposites in injection molding hyperbolical dies," Adv. Polym. Tech. 37, 3045-3055 (2018).

[42] Lee, K., and M. Mackley, "The significance of slip in matching polyethylene processing data with numerical simulation," J. Non-Newtonian Fluid Mech. 94, 159-177 (2000).

[43] Ansari, M., S. G. Hatzikiriakos, and E. Mitsoulis, "Slip effects in HDPE flows," J. Non-Newtonian Fluid Mech. 167, 18-29 (2012).

[44] Gleissle, W., and B. Hochstein, "Validity of the Cox-Merz rule for concentrated suspensions," J. Rheol. 47, 897-910 (2003).

[45] Mazzanti, V., F. Mollica, and N. El Kissi, "Rheological and mechanical characterization of polypropylene-based wood plastic composites," Polym. Compos. 37, 3460-3473 (2016).

[46] Köpplmayr, T., I. Milosavljevic, M. Aigner, R. Hasslacher, B. Plank, D. Salaberger, and J. Miethlinger, "Influence of fiber orientation and length distribution on the rheological characterization of glass-fiber-filled polypropylene," Polym. Test. 32, 535-544 (2013).

[47] Clemeur, N., R. Rutgers, and B. Debbaut, "Numerical simulation of abrupt contraction flows using the double convected Pom-Pom model," J. Non-Newtonian Fluid Mech. 117, 193-209 (2004).

[48] Hristov, V., E. Takacs, and J. Vlachopoulos, "Surface tearing and wall slip phenomena in extrusion of highly filled HDPE/wood flour composites," Polym. Eng. Sci. 46, 1204-1214 (2006).

[49] Ansari, M., S. G. Hatzikiriakos, A. M. Sukhadia, and D. C. Rohlfing, "Rheology of Ziegler-Natta and metallocene high-density polyethylenes: Broad molecular weight distribution effects," Rheol. Acta 50, 17-27 (2011).
[50] Duan, L., M. Spoerk, T. Wieme, P. Cornillie, H. Xia, J. Zhang, L. Cardon, and D. R. D'hooge, "Designing formulation variables of extrusion-based manufacturing of carbon black conductive polymer composites for piezoresistive sensing," Compos. Sci. Technol. 171, 78-85 (2019).

[51] Winter, H. H., "Three views of viscoelasticity for Cox-Merz materials," Rheol. Acta 48, 241-243 (2009).

[52] Comminal, R., F. Pimenta, J. H. Hattel, M. A. Alves, and J. Spangenberg, "Numerical simulation of the planar extrudate swell of pseudoplastic and viscoelastic fluids with the streamfunction and the VOF methods," J. Non-Newtonian Fluid Mech. 252, 1-18 (2018).

[53] Rueda, M. M., M. C. Auscher, R. Fulchiron, T. Perie, G. Martin, P. Sonntag, and P. Cassagnau, "Rheology and applications of highly filled polymers: A review of current understanding," Prog. Polym. Sci. 66, 22-53 (2017).

[54] Liang, J. Z., "Melt die-swell behavior of polyoxymethylene blended with ethylene-vinyl acetate copolymer and high-density polyethylene," Polym. Test. 68, 213-218 (2018).

[55] Béraudo, C., A. Fortin, T. Coupez, Y. Demay, B. Vergnes, and J. Agassant, "A finite element method for computing the flow of multimode viscoelastic fluids: Comparison with experiments," J. Non-Newtonian Fluid Mech. 75, 1-23 (1998).

[56] Ganvir, V., B. Gautham, H. Pol, M. S. Bhamla, L. Sclesi, R. Thaokar, A. Lele, and M. Mackley, "Extrudate swell of linear and branched polyethylenes: ALE simulations and comparison with experiments," J. Non-Newtonian Fluid Mech. 166, 12-24 (2011).

[57] Keunings, R., Simulation of Viscoelastic Fluid Flow (Hanser, New York, 1987).

[58] Spanjaards, M., M. Hulsen, and P. Anderson, "Transient 3D finite element method for predicting extrudate swell of domains containing sharp edges," J. Non-Newtonian Fluid Mech. 270, 79-95 (2019).

[59] See supplementary material at https://doi.org/10.1122/1.5145255 for the geometry, the mesh patterns, the extra rheological data for neat HDPE and LDPE melts, the extrudate swell data for PP, the pressure profiles with the 3D model, and the nonisothermal simulation results (Figs. S1-S10). In addition, the raw data on pressure measurements, the PTT material parameters for neat PP, and the extra parameters for nonisothermal modeling are shown in Tables S1-S3. 